\title{
Elections législatives 2007
}

Cartes électorales et instrumentalisation de la refondation de la gauche

\section{Céline Colange et Michel Bussi}

\section{(2) OpenEdition}

Journals

Édition électronique

URL : http://journals.openedition.org/echogeo/1185

DOI : 10.4000/echogeo.1185

ISSN : 1963-1197

\section{Éditeur}

Pôle de recherche pour l'organisation et la diffusion de l'information géographique (CNRS UMR 8586)

Référence électronique

Céline Colange et Michel Bussi, «Elections législatives 2007 », EchoGéo [En ligne], Sur le Vif, mis en ligne le 21 août 2007, consulté le 04 mai 2019. URL : http://journals.openedition.org/echogeo/1185 ; DOI : 10.4000/echogeo. 1185

Ce document a été généré automatiquement le 4 mai 2019.

\section{(c) (i) () $\Theta$}

EchoGéo est mis à disposition selon les termes de la licence Creative Commons Attribution - Pas d'Utilisation Commerciale - Pas de Modification 4.0 International 


\title{
Elections législatives 2007
}

Cartes électorales et instrumentalisation de la refondation de la gauche

\author{
Céline Colange et Michel Bussi
}

\section{NOTE DE L'ÉDITEUR}

Avec la participation de Jean-Paul Gosset

1 La carte électorale est un outil commode et relativement consensuel dans le jeu des analyses post-électorales. Le lendemain des scrutins, elle autorise des descriptions exhaustives des comportements électoraux, et met d'ailleurs souvent en évidence des permanences, qui relativisent les vagues décrites par les médias nationaux ou les particularismes inventoriés par les médias locaux. La carte électorale, notamment depuis l'ère informatique, devance l'exploitation des sondages post-électoraux: c'est donc également la carte électorale qui, comparée à partir d'une approche "écologique ${ }^{1}$ à d'autres cartes (scrutins précédents, données socio-économiques), permet de lancer les premières hypothèses explicatives... Nous proposons dans cet article non seulement d'analyser les résultats électoraux des élections législatives de 2007, mais également de débattre des interprétations politiques possibles de la carte, par exemple au regard de certaines analyses médiatiques (Le Bras, Levy, 2007).

2 A priori, les élections législatives 2007 ne semblaient pas forcément "passionnantes » pour les amateurs de cartes électorales. Ces élections apparaissaient comme la confirmation attendue de l'élection présidentielle, et de la « nouvelle " géographie qu'elle révéla ${ }^{2}$, la mobilisation citoyenne en moins. Pourtant, presque contre toute attente, l'analyse des cartes des élections législatives oblige à poser un problème fondamental de rapport entre la carte électorale et la stratégie politique.

Pour simplifier, les élections législatives de 2007 furent marquées au premier tour par un effondrement du Front national, qui fut globalement associé à l'augmentation du score de l'UMP ; au second tour, le « sursaut » de la gauche fut associé au report massif du Modem vers le parti socialiste. Dans ce sens, Hervé Le Bras ${ }^{3}$ (Libération, 20 Juin 2007) n'hésite pas à utiliser une métaphore empruntée à la géographie physique : «la droite s'étant approchée 
de l'extrême droite, elle s'est éloignée de centre, qui occupe l'espace ainsi libéré. C'est d'ailleurs le mécanisme qui produit les fameux tsunamis: une plaque continentale glisse vers une autre, ouvrant une faille où l'eau s'engouffre. La plaque UMP s'est rapprochée de la plaque FN. Dans la faille se glisse alors un nouveau centre fait de chiraquiens anti-FN, d'écologistes réticents à l'alliance systématique avec la gauche et de républicains modérés, soit une clientèle qui écarte un peu plus la gauche de ses racines populaires ".

4 La carte ainsi décryptée n'est plus seulement utile à l'analyse, elle devient l'instrument d'une stratégie politique, comme le confirme Hervé Le Bras (2007) : "L'avenir du parti socialiste est dessiné par cette dynamique: soit le socialisme se replie sur son idéologie et visiblement, il vivotera dans ses bastions traditionnels progressivement grignotés comme le sont ceux du PC. Soit il accordera de l'attention aux demandes dont sont porteurs ces nouveaux courants discrets qui se profilent en terre de droite... ». Jacques Lévy', (2007), à partir d'une analyse cartographique proche de celle d'Hervé Le Bras (progression de la gauche dans les hyper centres des grandes métropoles, stagnation dans les foyers traditionnels), instrumentalise lui aussi la carte électorale pour proposer une nouvelle donne partisane : " pour se refonder, la gauche doit d'abord solder la part conservatrice de l'héritage du mouvement ouvrier et assumer la complémentarité de ce qui, dans la vieille gauche et la vieille droite, est présenté comme la contradictoire: ordre et justice, liberté et responsabilité, innovation et solidarité, identité et cosmopolitisme ».

5 Ce glissement de la carte électorale vers la prospective partisane peut apparaître scientifiquement discutable. D'une part, il existe plusieurs cartes d'évolutions des résultats électoraux possibles et plusieurs interprétions acceptables de ces cartes. D'autre part, il est dangereux de passer directement d'une superposition de cartes à des comportements individuels supposés, puis de ces comportements individuels supposés à une stratégie partisane unique. Après avoir analysé plus précisément le fait majeur de ces élections, l'effondrement du Front national, nous analyserons, à partir de l'évolution 2002-2007 du score de la gauche et de la droite, la complexité des recompositions en cours.

Vers la fin du Front national?

6 Avec seulement $4,29 \%$ des suffrages exprimés au premier tour de ce scrutin législatif du printemps 2007, le Front national réalise son plus mauvais score depuis sa percée électorale lors des européennes de 1984. En raison de la contre-performance de JeanMarie Le Pen à la présidentielle, le reflux de l'extrême droite semblait prévisible mais personne ne s'attendait à une telle ampleur du phénomène. Marine Le Pen, la fille du leader, est la seule candidate frontiste à se qualifier pour le second tour. Parmi les autres personnalités du parti qui tentaient de briguer un mandat parlementaire, Carl Lang (dans la $23^{e}$ circonscription du Nord: Maubeuge) et Bruno Gollnisch (dans la $13^{e}$ circonscription du Rhône : Meyzieu) essuient eux aussi un sérieux revers. Ils obtiennent respectivement $11,60 \% 6,95 \%$ des voix dans des terrains pourtant socialement et économiquement favorables, où le Front national obtenait autrefois près de $20 \%$ des suffrages. Le recul du parti de Jean-Marie Le Pen est donc significatif en comparaison avec la situation observée lors des précédentes élections de ce type en 2002 ou en 1997. Le Front national était alors présent au second tour dans 132 circonscriptions, dont 76 triangulaires, favorisant ainsi l'élection de plusieurs députés de gauche.

L'effritement du Front national est net dans la partie septentrionale du pays, des côtes normandes à l'Alsace, en passant par le sillon mosellan, les plaines champenoises et ardennaises, le bassin parisien ( $\mathrm{du}$ sud du département de l'Eure jusqu'aux frontières 
picardes de l'Oise et de l'Aisne), le département du Nord et enfin le midi méditerranéen, de Perpignan à Cannes, et son arrière-pays (Vaucluse, basses-Alpes) (figure 1). Ces circonscriptions correspondent à des espaces devenus progressivement des bastions frontistes au cours des décennies 1980 et 1990. Cependant, la carte montre que l'érosion est atténuée dans le nord-est, en particulier en Lorraine, dans la Haute-Marne et le dans Législatives 2007 - Premier tour Evolution du Front National entre 2002 - 2007 (en pourcentage des suffiages exprimés)

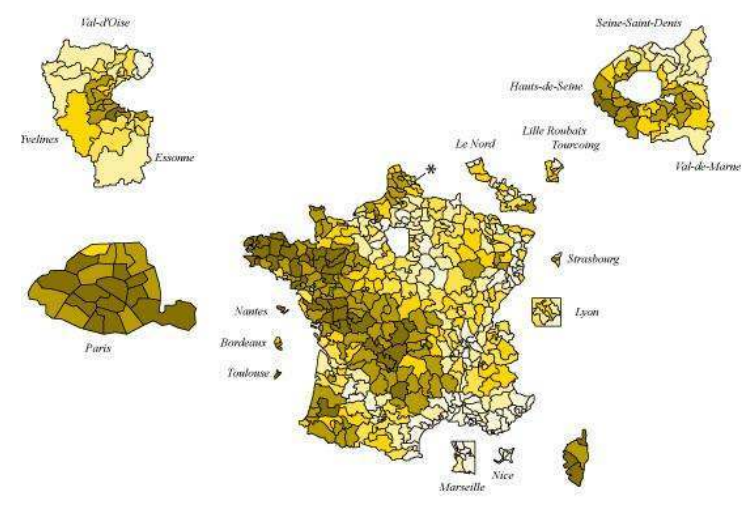

le Pas-de-Calais.

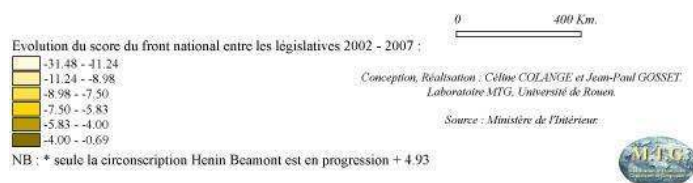

Mécaniquement, les pertes sont donc plus importantes là où le Front national comptabilisait le plus grand nombre de suffrages. A l'inverse, le recul apparaît beaucoup moins marqué dans les régions qui ont toujours exprimé une résistance à ce parti, mais où celui-ci avait davantage progressé en 2002, notamment en Bretagne, dans les Pays-de-la Loire, le Poitou, le Limousin ou la Corse.

7 Le transfert de vote d'une partie de l'électorat Front national vers l'UMP mis en lumière par les cartes et les analyses des résultats de la présidentielle semble se confirmer. Néanmoins, il s'agit d'un phénomène bien plus complexe qu'une simple machine mécanique fonctionnant selon un système de vases communicants. La stratégie de Nicolas Sarkozy qui est allé «chassé » sur les terres de l'extrême droite en développant et en véhiculant un discours de fermeté sur les thèmes de la sécurité, de l'immigration et de «l'identité nationale » s'est avérée néfaste pour le Front national. Jean-Marie Le Pen n'est pas parvenu à reconquérir la frange de son électorat séduite par le programme du Président nouvellement élu, mais surtout, il n'a pas réussi à stopper l'hémorragie électorale subie par son parti. Désormais, le Front national ne compte plus dans ses rangs que les lepénistes de la première heure. Il n'est pas parvenu à fidéliser véritablement les électeurs conquis ces vingt dernières années. Par son discours libéral «décomplexé » opposé au « gaullisme social » de Jacques Chirac, Nicolas Sarkozy a ramené au sein de son camp politique d'anciens électeurs de la droite qui s'étaient radicalisés et votaient massivement pour le Front national.

8 La tripartition de l'espace politique évoquée par les politistes à la fin des années 1990 s'efface au profit d'un renforcement du bipartisme et de la bipolarisation. L'appareil 
politique national est désormais dominé par deux grandes familles : l'UMP à droite et le parti socialiste à gauche. Le Front national qui jouait autrefois un rôle d'arbitre majeur pour la victoire ou l'obtention d'une majorité électorale semble aujourd'hui marginalisé. Les déchirements au sein du Front national font apparaître Jean-Marie Le Pen comme un chef de file vieillissant sur la scène politique nationale face à une garde de «jeunes loups » débordant d'ambitions. Tant que Nicolas Sarkozy tiendra les rennes du pouvoir au sein de son propre camp, le Front national restera très probablement « en sommeil ». Toutefois, on peut supposer que la moindre faille du président Sarkozy ravivera l'étincelle de l'extrême droite, à moins que les querelles internes au parti pour succéder au "père fondateur " n'éteignent définitivement le «flambeau tricolore », symbolisant du Front national.

De la carte électorale à la refondation de la gauche

La carte d'évolution de la droite entre 2002 et 2007 confirme l'hypothèse du transfert des voix frontistes vers les candidats de l'UMP (figure 2). Les progrès de l'UMP sont particulièrement nets dans l'est, notamment pour l'Alsace, le littoral méditerranéen, les marges du bassin parisien. Cette évolution, logiquement, est très proche de celle de Nicolas Sarkozy vis-à-vis du vote pour Jacques Chirac en 2002, et confirme des analyses Législatives 2007 - Premier tour
Evolution de la droite entre 2002 - 2007 (en pourcentage des suffrages exprimés)

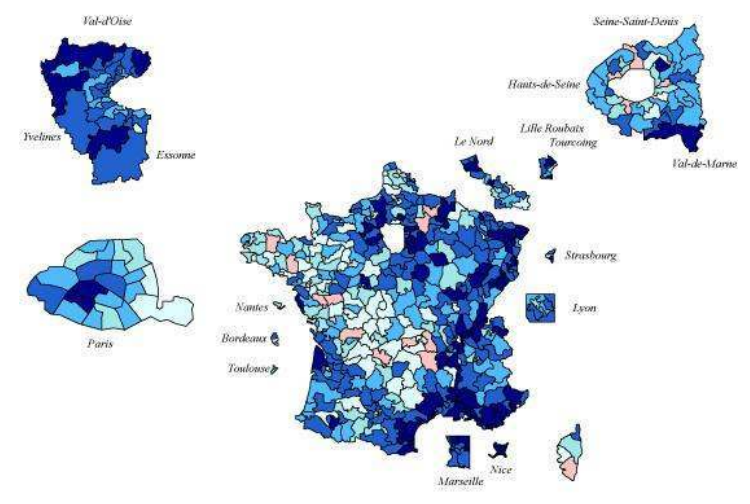

antérieures ${ }^{5}$.

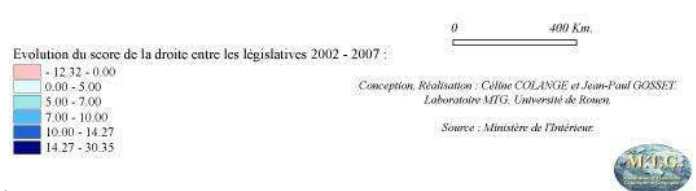

La carte d'évolution de la gauche entre 2002 et 2007 est plus complexe à analyser. En pourcentage des suffrages exprimés, la gauche semble avoir progressé dans trois types de 

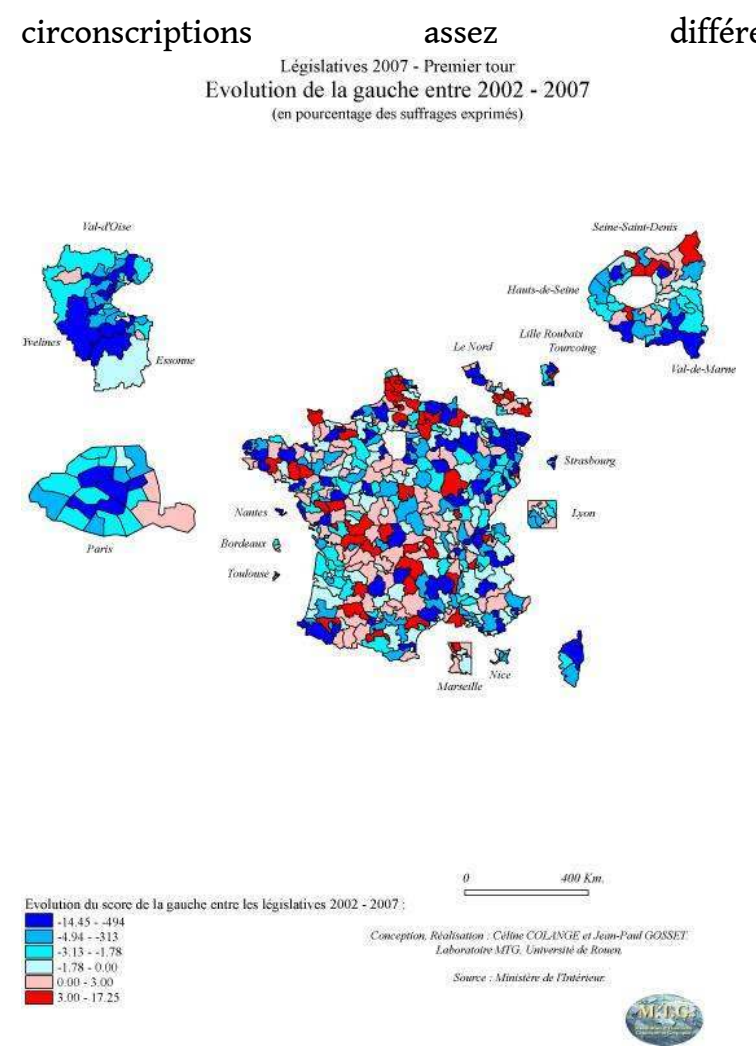

L'évolution positive dans le cœur des grandes agglomérations se vérifie, sans être flagrante: une petite dizaine de circonscriptions de centre ville basculent pour la première fois à gauche: Paris 8 , Lyon 2 et 3, Marseille 4, Bordeaux 2, Toulouse 1... Il s'agit cependant d'îlots assez isolés. La gauche avait déjà progressé en 2002 dans ces espaces (suite notamment aux conquêtes municipales de 2001). Le constat « urbain » principal reste tout de même les progrès de l'UMP depuis 2002 dans les espaces les plus privilégiés, comme c'est le cas dans la plupart des circonscriptions parisiennes (notamment les 1, 2, 4, 6) ou de Lyon, Nantes, Strasbourg, Lille...

L'évolution de la gauche semble beaucoup plus évidente dans les circonscriptions habituellement qualifiées de "populaires » : l'effet est net pour l'espace Somme-AisneNord-Pas de Calais, où vraisemblablement, la gauche bénéficie elle aussi d'une partie de l'effondrement du Front national. La progression est également importante en SeineSaint-Denis ou dans les Bouches-du-Rhône. On peut y voir à la fois « l'effet de traîne » de la forte mobilisation anti-Sarkozy dans les quartiers populaires (marquée notamment par une participation massive, qui s'est effondrée en un mois), et peut-être la capacité de la gauche à récupérer elle aussi l'électorat populaire et ouvrier qui votait Front national depuis près de 20 ans, lorsqu'elle dispose d'un fort réseau d'élus, d'associations ou de militants locaux.

Enfin, l'évolution de la gauche est particulièrement net dans l'Ouest, dans une France " apaisée ${ }^{6}$. Les terres radicales du sud Ouest restent à gauche sans progression majeure. Le centre Ouest de la France (Poitou-Limousin), pris en «tenaille» par les aires d'influences de l'ex-couple Royal-Hollande, apparait comme une évidente terre de conquête, dans un contexte de post-Chiraquie. Le score de la gauche est également en évolution positive depuis cinq ans dans de nouveaux espaces bretons (Morbihan, Ille et Vilaine), et de façon plus inattendue en Basse-Normandie. Dans ces circonscriptions, outre les bons scores des Ségolène Royal lors des présidentielles, la gauche semble 
bénéficier du travail de proximité effectué par les élus de gauche depuis 2001 et 2004, à la suite du basculement de nombreuses collectivités locales (départements, régions). Outre le contrôle du pouvoir local, la gauche peut y présenter des candidats connus et actifs. Cette explication est rarement retenue par les analystes à l'échelle nationale, alors que dans un contexte de décentralisation accrue, le poids des contextes politiques de proximité et des pouvoirs locaux réels des candidats est souvent important, notamment pour des électeurs de moins en moins sensibles aux débats idéologiques.

12 Au regard de cette diversité de situation, la «tectonique» des plaques PC-PS-Modem décrite par Hervé Le Bras (et basée sur la cartographie de l'évolution de la gauche entre le premier et le second tour des législatives 2007), n'apparaît pas comme une clé de lecture unique. De même, l'analyse géographique «urbano-centrée» de Jacques Lévy, et son implication politique (un parti socialiste qui doit se pencher davantage vers le Modem que le $\mathrm{PC}$ ), tient davantage de la mobilisation d'une carte pour soutenir une position idéologique, que de l'inverse. Les terres «rurales" radicales et les bassins industriels continuent de fournir les plus gros bataillons de députés à la gauche, notamment à travers des accords locaux sans faille entre élus socialistes et communistes. Le grand écart auquel se trouve confronté le parti socialiste (des «bobos» aux «cocos») est de fait proche de celui que doit résoudre l'UMP, qui a progressé dans les espaces les plus privilégiés (ceux qui ont le plus «à gagner »), tout en ayant pour base électorale ceux qui se vivent comme relégués ou oubliés (dont l'idéal-type reste le ménage périurbain).

En terme de géographie électorale, le glissement d'artefacts collectifs (les résultats des 577 circonscriptions électorales) à une injonction politique: «ce que peuvent faire les socialistes : écouter une société d'individus en mouvement $»^{7}$ est sans doute un peu rapide. Les individus en mouvement (les urbains à fort capital spatial qui maîtrisent leur mobilité ?) représentent-ils davantage l'avenir du nouveau «peuple » d'une gauche refondée, que les électeurs captifs des quartiers de la politique de la ville, que les salariés des bassins d'emploi en reconversion économique, que les navetteurs relégués des espaces périurbains ? On peut aussi faire l'hypothèse inverse que les progrès de la gauche dans les hyper centres (autant de confettis sur la carte de France) sont l'illustration cartographique d'une gauche coupée de sa base sociale, et que pour reconquérir le pouvoir, la gauche, et le parti socialiste en particulier, devra sortir des faubourgs.

\section{BIBLIOGRAPHIE}

Bussi M., Colange C., Gosset J.P., 2007, « Elections présidentielles 2007, typologie des candidats. Les deux France », revue électronique Cybergéo, rubrique Points Chauds.

Bussi M., Colange C., Gosset J.P., 2007, « Présidentielles 2007 second tour : Nouvelle géographie des votes et réalignement partisan », revue électronique Cybergéo, rubrique Points Chauds.

Bussi M., Colange C., Gosset J.P., 2007, « Elections présidentielles, 1er tour 2007, nouveaux candidats nouvelle géographie des votes? (une analyse de l'évolution 2002-2007 par canton), rubrique actualité de Géoconfluences. 
Colange C., 2007, « Réalignements et désalignements du vote en France : 1981-2005 », thèse de doctorat sous la direction de Monsieur Michel Bussi, Université de Rouen, 350 p.

Le Bras H., 2007, « Les électeurs se sont reportés à haut débit sur le PS », Libération, 20 juin 2007, p. 12.

Lévy J., 2007, « Rose en deçà des faubourgs, bleu au-delà », Libération, 20 juin 2007, p. 13.

\section{NOTES}

1. L'approche écologique ne s'intéresse pas aux électeurs eux-mêmes mais aux entités spatiales dans lesquelles ils évoluent (leur environnement géographique). Cette méthode consiste à comparer des données agrégées au sein d'unités territoriales, notamment à l'aide de cartes. Cette approche a néanmoins des limites dont la plus connue est l'erreur écologique liée à la signification des corrélations, visuelles ou statistiques.

2. Bussi Michel, Colange Céline, Gosset Jean-Paul, « Elections présidentielles 2007, typologie des candidats. Les deux France »; « Présidentielle 2007 second tour : Nouvelle géographie des votes et réalignement partisan »; rubriques points chauds de Cybergéo. 3. Le Bras Hervé, «Les électeurs se sont reportés à haut débit sur le PS », Libération, 20 juin 2007, p. 12.

4. Lévy Jacques, « Rose en deçà des faubourgs, bleu au-delà », Libération, 20 juin 2007, p. 13.

5. Bussi Michel, Colange Céline, Gosset Jean-Paul, « Élections présidentielles, 1er tour 2007, nouveaux candidats, nouvelle géographie des votes? (une analyse de l'évolution 2002-2007 par canton) », rubrique actualité de Géoconfluences.

6. Bussi Michel, Colange Céline, Gosset Jean-Paul, « Elections présidentielles 2007, typologie des candidats. Les deux France »; rubriques points chauds de Cybergéo.

7. J. Lévy, conclusion de l'article « Rose en deçà des faubourgs, bleu au-delà », Libération, 20 juin 2007, p. 13.

\section{RÉSUMÉS}

Cette publication s'appuie sur l'étude cartographique des résultats du premier tour des élections législatives du mois de juin 2007 à l'échelle des 577 circonscriptions de la métropole. Outre la description et la compréhension de la dimension spatiale des votes, cet article propose quelques pistes de réflexion sur les interprétations de la carte électorale et sur son instrumentalisation possible par les médias nationaux ou locaux. En particulier, les propositions pour une refondation de la gauche seront différentes selon que l'on mette en évidence l'évolution de la gauche dans les hyper centres urbains, dans les campagnes ouvrières de l'ouest, dans les banlieues urbaines ou dans les bassins industriels. 